\title{
Influence of iron- and zinc-chelating agents on neutrophil extracellular trap formation
}

\author{
WERONIKA KUŹMICKA ${ }^{1,2}$, ANETA MOSKALIK', ANETA MANDA-HANDZLIK', \\ URSZULA DEMKOW ${ }^{1}$, MAEGORZATA WACHOWSKA ${ }^{1}$, OLGA CIEPIELA ${ }^{3}$
}

${ }^{1}$ Department of Laboratory Diagnostics and Clinical Immunology of Developmental Age, Medical University of Warsaw, Warsaw, Poland ${ }^{2}$ Postgraduate School of Molecular Medicine, Medical University of Warsaw, Warsaw, Poland ${ }^{3}$ Department of Laboratory Medicine, Medical University of Warsaw, Warsaw, Poland

\begin{abstract}
Release of neutrophil extracellular traps (NETs) is one of the neutrophils' mechanisms involved in the response to infection. NETs are released from the cell in response to a biological or synthetic stimulus to entrap, immobilize and kill pathogens. Metal ions and metal binding proteins were identified in the structure of NETs, but their role in NET release remains unclear. The aim of this study was to assess how lack of iron and zinc generated by ion sequestration using chelators affects NET release. Neutrophils were isolated from whole blood or buffy coats of healthy blood donors by density gradient centrifugation and incubated with zinc chelators: $20 \mu M N, N, N^{\prime}, N^{\prime}$-Tetrakis(2-pyridylmethyl)ethylenediamine (TPEN), $40 \mu M$ diethylenetriaminepentaacetic acid (DTPA) or iron chelators: $400 \mu M$ deferoxamine mesylate salt (DFO) and $50 \mu \mathrm{M}$ iminodiacetic acid (IDA). Next, $100 \mathrm{nM}$ phorbol 12-myristate 13-acetate (PMA) was added to stimulate release of NETs. The amount of released DNA was measured by fluorometry and NETs were visualized by immunofluorescence microscopy. This study demonstrates that iron and zinc chelators are able to modulate NET release. Here we show that preincubation of neutrophils with TPEN and IDA inhibits NET release in cells stimulated with PMA. On the other hand, DFO stimulates NET release. Incubation of cells with DTPA does not affect release of NETs.
\end{abstract}

Key words: deferoxamine, iminodiacetic acid, DTPA, TPEN, neutrophil extracellular traps.

(Cent Eur J Immunol 2021; 46 (2): 135-139)

\section{Introduction}

Neutrophils represent the most numerous group of leukocytes in peripheral blood of adult humans. They play an essential role in the innate immune response and are the first pillar of defense against invading pathogens. Neutrophils employ diverse strategies during an infection: phagocytosis, degranulation, release of reactive oxygen species (ROS) and release of neutrophil extracellular traps (NETs). NET release is a process leading to extracellular release of threads of decondensed chromatin combined with biocidal proteins to fight and kill pathogens. NET release can be triggered by biological inducers such as bacteria, parasites, fungi and viruses or non-physiological stimuli: phorbol 12-myristate 13-acetate (PMA) and calcium ionophore. To date, it is considered that there are two processes of critical importance for NET release: synthesis of ROS and histone citrullination [1-5]. NETs play a crucial role in the maintenance of immune homeostasis as their insufficient or excessive production can lead to autoimmune disorders, diabetes or cancer [6].

Numerous studies highlight the essential interrelation of microelements with the innate immune response $[7,8]$.
However, the exact connection between metal ions and NET release remains unclear. Microelements are necessary not only for humans, but also for invading pathogens which scavenge them from the infected host to survive. Consequently, every infection becomes a battlefield for microelements. Moreover, ion disorders in the body can contribute to immune homeostasis imbalance and consequently to a lost battle during infection for the human [7, 9-11]. According to the latest findings, NETs incorporate metallic ions and contain zinc and iron binding proteins as well [12]. However, to this day, little is known about the exact role of these ions in NET formation and what the effect of their sequestration will be. Therefore, we aimed to provide new insight in the field by exploring the effect of zinc and iron chelating agents on a crucial type of innate immunity response - the process of NET formation.

\section{Material and methods}

\section{Neutrophil isolation}

Buffy coats or venous blood of healthy blood donors were collected into citrate tubes and purchased from the

Correspondence: Olga Ciepiela, PhD, Department of Laboratory Medicine, Medical University of Warsaw, Banacha 1 A, 02-097 Warsaw, Poland, e-mail: olga.ciepiela@wum.edu.pl

Submitted: 19.01.2021; Accepted: 3.03.2021 
Regional Center for Blood Donation and Blood Treatment in Warsaw. Cells were isolated by density gradient centrifugation as described previously [13]. Next, the diluted cell mass was layered on Histopaque 1077 (Ref. no.: 10771, Sigma Aldrich, St. Louis, MO, USA). Samples were centrifuged and then mononuclear cells with Histopaque 1077 were discarded. Remaining red blood cells and neutrophils were gently mixed with $1 \%$ polyvinyl alcohol and left for erythrocyte sedimentation. Residual erythrocytes were lysed with water. Neutrophils were washed and resuspended in RPMI 1640 without phenol red medium supplemented with $10 \mathrm{mM}$ HEPES (Ref. no.: 17-737E, Lonza, Basel, Switzerland).

\section{NETs quantification}

Neutrophils $\left(5 \times 10^{4}\right.$ cells/well $)$ were treated with $20 \mu \mathrm{M} N, N_{,} \mathrm{N}^{\prime}, \mathrm{N}^{\prime}$-Tetrakis(2-pyridylmethyl)ethylenediamine (TPEN, Sigma-Aldrich, St. Louis, MO, USA), $40 \mu \mathrm{M}$ diethylenetriaminepentaacetic acid (DTPA, Sigma-Aldrich, St. Louis, MO, USA), $400 \mu \mathrm{M}$ deferoxamine mesylate salt (DFO, Sigma-Aldrich, St. Louis, MO, USA) or $50 \mu \mathrm{M}$ iminodiacetic acid (IDA, Sigma-Aldrich, St. Louis, MO, USA) for $30 \mathrm{~min}$ at $37^{\circ} \mathrm{C}, 5 \% \mathrm{CO}_{2}$ in 24-well plates. Then 100 nM PMA (Ref. no.: P1585, Sigma Aldrich, St. Louis, MO, USA) was added to the cells to stimulate NET release for $3 \mathrm{~h}$ at $37^{\circ} \mathrm{C}$, $5 \% \mathrm{CO}_{2}$. Untreated cells served as a control. Subsequently, extracellular DNA was detached from the wells' bottom with $10 \mathrm{U} / \mathrm{ml}$ deoxyribonuclease (Ref. no.: DN25, Sigma-Aldrich, St. Louis, MO, USA). The process was stopped with $5 \mathrm{mM}$ EDTA, plates were centrifuged and supernatant was collected. After that, 100 nM SYTOX Green (Life Technologies, Carlsbad, California, USA) was added and the amount of extracellular DNA was quantified by fluorometry with a FLUOstar OMEGA plate reader (BMG Labtech, Ortenberg, Germany).

\section{NETs visualization}

The cells $\left(2.5 \times 10^{4}\right.$ cells/well $)$ were seeded into LabTek chamber cover slides and incubated with the studied chelators or medium alone for $30 \mathrm{~min}$ at $37^{\circ} \mathrm{C}, 5 \% \mathrm{CO}_{2}$. Next, 100 nM PMA was added to stimulate NET release for $3 \mathrm{~h}$ at $37^{\circ} \mathrm{C}, 5 \% \mathrm{CO}_{2}$. Then, samples were fixed with $4 \%$ paraformaldehyde, permeabilized with $0.1 \%$ Triton $\mathrm{X}$ (Sigma-Aldrich, St. Louis, MO, USA) and stained overnight with anti-myeloperoxidase-FITC monoclonal antibody (1: 500, $4^{\circ} \mathrm{C}$, Abcam ab11729, Cambridge, UK). DNA was counterstained with $1 \mu \mathrm{M}$ SYTOX Orange (Life Technologies, Carlsbad, California, USA). NET visualization was performed with a Leica DMi8 fluorescence microscope (Leica, Wetzlar, Germany) equipped with a $40 \times$ and a $10 \times$ magnification objective.

\section{Statistical analysis}

Statistical analysis was carried out using GraphPad Prism 8 (GraphPad Software, La Jolla, California, USA). Normality of distribution was analyzed by the Shapiro-Wilk test. All values were analyzed with one-way ANOVA followed by an appropriate post-hoc test. All the results are presented as mean \pm standard error of the mean. The results were considered statistically significant at a $p$-value $\leq 0.05$.

This study was approved by the Ethical Committee at Medical University of Warsaw, no. KB/92/2017. Informed written consent was obtained from volunteer blood donors.

\section{Results}

\section{Intracellular zinc chelation inhibits NET release}

We studied the effect of zinc chelation on NET release using TPEN, an intracellular zinc chelator and DTPA, an extracellular zinc chelator. Quantitative analysis and fluorescent microscopy indicated that incubation of neutrophils with TPEN inhibits NET release stimulated with PMA (Fig. 1A, C). Interestingly, pretreatment of cells with DTPA had no effect on neutrophils' ability to release NETs (Fig. 1B, C). Neither studied compound affected NET release when used alone (Fig. 1A-C).

\section{Iron chelators affect release of NETs}

We decided to investigate how different iron chelators - DFO and IDA - affect release of NETs. In our hands, pretreatment of cells with DFO stimulated neutrophils to release NETs. However, DFO had no effect on PMA-stimulated NET release (Fig. 2A). Moreover, we found that preincubation of cells with IDA caused a significant decrease in NET release after stimulation with PMA. IDA alone did not affect neutrophils' ability to release NETs (Fig. 2B). Fluorescent microscopy confirmed the above results (Fig. 2C).

\section{Discussion}

In our study we aimed to investigate how iron and zinc sequestration affects neutrophils' ability to release NETs. Our results demonstrated that zinc chelation by the intracellular chelator TPEN inhibits release of NETs. Moreover, the iron chelators deferoxamine and iminodiacetic acid affect NET release, although each one in a different way.

Iron chelators are compounds applied in iron poisoning and during treatment of cancer or thalassemia $[14,15]$. In our study we analyzed the potent drug DFO. DFO has been reported to cause immunological changes by increasing the total number of lymphocytes as well as natural killer cells, 

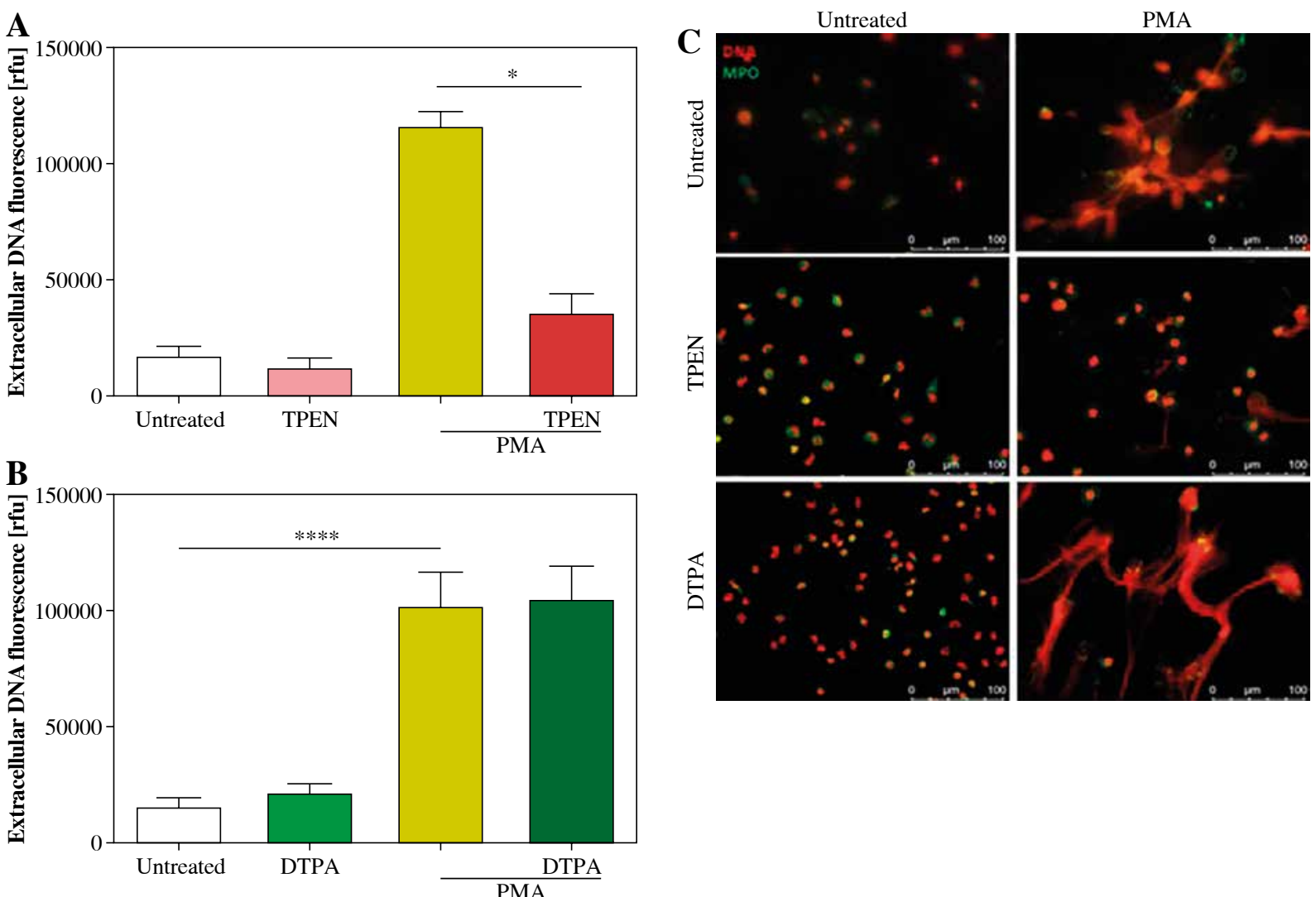

Fig. 1. Depletion of intracellular zinc inhibits NET release. Neutrophils were incubated with A) $20 \mu \mathrm{M}$ TPEN or B) $40 \mu \mathrm{M}$ DTPA. Untreated cells served as a control. Next, $100 \mathrm{nM}$ PMA was added to stimulate release of NETs. Extracellular DNA release was $(\mathbf{A}, \mathbf{B})$ quantified with fluorometry and $(\mathbf{C})$ visualized with fluorescent microscopy. A, B) Data are shown as means \pm SEM and were analyzed with one-way ANOVA with post-hoc (A) Dunn's or (B) Dunnett's test. A, B) $n=6$. ${ }^{*} p \leq 0.05$, **** $p \leq 0.0001$. MPO - myeloperoxidase

CD4, CD8 and immunoglobulin E (IgE) serum levels [16]. Our study demonstrated that iron sequestration with a dose of $400 \mu \mathrm{M}$ DFO induced release of NETs. Our outcome is consistent with results obtained by Völlger et al., who reported that induction of NETs by DFO requires nicotinamide adenine dinucleotide phosphate-dependent ROS production, peptidylarginine deiminase 4 activation and proteases [17]. On the other hand, Kono et al. reported that another iron chelator - deferasirox (DFS) - inhibits cellular vacuolation and release of NETs [18]. These findings are constant with Saigo et al., who reported that DFS inhibits ROS production in neutrophils incubated with PMA [19]. Taking into consideration different effects of each of the two aforementioned iron chelators, we suggest that there are different mechanisms of action on neutrophils depending on the applied compound.

In our study we also analyzed another iron chelating compound, IDA, which was reported to chelate ferric iron. Interestingly, the complex Fe(III)-IDA was reported to be applied to enable the delivery of iron into the cells in order for them to grow and differentiate [20]. In our study, we demonstrated that iron sequestration with IDA does not affect neutrophils. Curiously, cells preincubated with IDA and stimulated with PMA possess a weakened capacity to release NETs.

Changes in zinc intracellular concentration were reported during NET release [21]. Moreover, zinc is vital for proper activity of calprotectin, which is an antimicrobial protein stored in neutrophilic granules and a component of NET structures [22]. We previously demonstrated that incubation of neutrophils with zinc inhibits NET release by affecting citrullination of histone H3 [23]. Here we demonstrate that zinc intracellular depletion by the intracellular chelator TPEN inhibits NET release. Furthermore, zinc sequestration from the medium did not alter release of NETs. Our results demonstrate that zinc stored inside the cell and not supplied with the medium is involved in the release of NETs. Interestingly, zinc chelation was reported to impair neutrophils' functions, including release of ROS, degranulation and phagocytosis [24]. Considering 
A

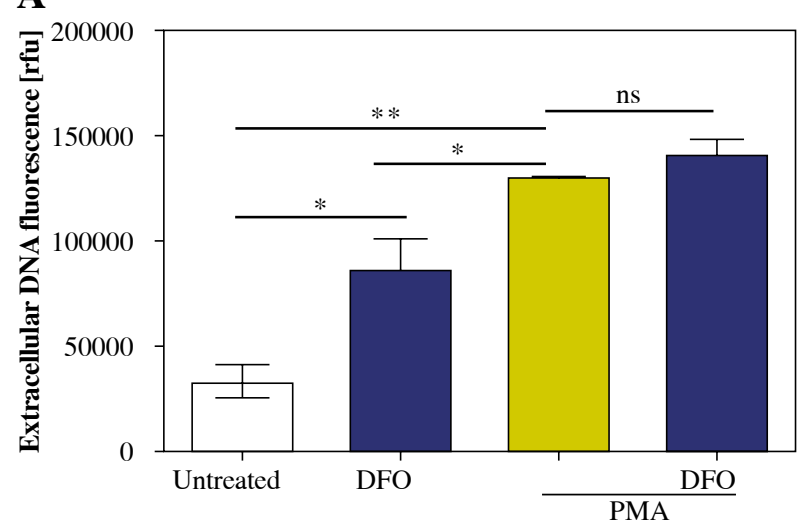

C

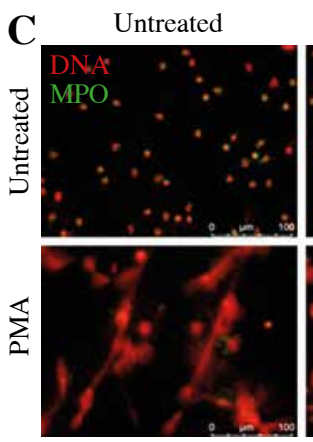

DFO

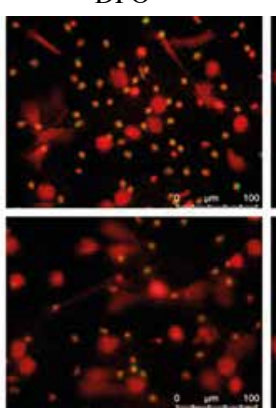

IDA

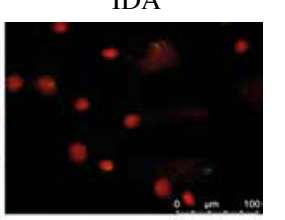

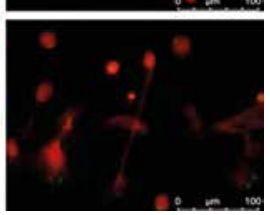

B



Fig. 2. Iron chelators affect neutrophils' ability to release NETs. Isolated cells were treated with A) $400 \mu \mathrm{M}$ DFO or B) $50 \mu \mathrm{M}$ IDA for 30 minutes. Next, NET release was stimulated with $100 \mathrm{nM}$ PMA. Amount of released DNA was analyzed with (A) fluorometry and (B) fluorescent microscopy. Data are shown as means \pm SEM and were analyzed with one-way ANOVA with post-hoc (A) Tukey's or (B) Sidak's test. A) $n=3$, B) $n=5$. $* p \leq 0.05, * * p \leq 0.01$. MPO - myeloperoxidase

that histone citrullination and ROS release can be affected by different levels of intracellular zinc concentrations and that these processes are vital for NET release, we suggest that an optimal intracellular zinc concentration is required for proper release of NETs.

In conclusion, this study demonstrates that iron and zinc chelators are able to impair innate immunity by modulating neutrophils' ability to release NETs. However, it should be acknowledged that some iron chelators may themselves provoke neutrophils to release NETs. Furthermore, inadequate zinc levels may inhibit neutrophils' capacity to release NETs. Future more detailed investigations of the mechanisms in which iron and zinc sequestration influences NET release are warranted.

\section{Funding}

This study was supported by the Students' Mini-Grant No. 1WW/NM1/16 funded by the Dean of the First Faculty of Medicine, Medical University of Warsaw (A.M.) and National Science Centre, Poland; Preludium grant no. 2017/25/N/NZ6/00142 (W.K., U.D.).

The authors declare no conflict of interest.

\section{References}

1. Brinkmann V, Reichard U, Goosmann C, et al. (2004): Neutrophil extracellular traps kill bacteria. Science 303: 1532-1535.

2. Delgado-Rizo V, Martinez-Guzman MA, Iniguez-Gutierrez L, et al. (2017): Neutrophil extracellular traps and its implications in inflammation: an overview. Front Immunol 8: 81.

3. Remijsen Q, Vanden Berghe T, Wirawan E, et al. (2011): Neutrophil extracellular trap cell death requires both autophagy and superoxide generation. Cell Res 21: 290-304.

4. Selders GS, Fetz AE, Radic MZ, Bowlin GL (2017): An overview of the role of neutrophils in innate immunity, inflammation and host-biomaterial integration. Regen Biomater 4: 55-68.

5. White PC, Chicca IJ, Ling MR, et al. (2017): Characterization, quantification, and visualization of neutrophil extracellular traps. Methods Mol Biol 1537: 481-497.

6. Sorensen OE, Borregaard N (2016): Neutrophil extracellular traps - the dark side of neutrophils. J Clin Invest 126: 16121620.

7. Hood MI, Skaar EP (2012): Nutritional immunity: transition metals at the pathogen-host interface. Nat Rev Microbiol 10: 525-537.

8. Maares M, Haase H (2016): Zinc and immunity: an essential interrelation. Arch Biochem Biophys 611: 58-65.

9. Cassat JE, Skaar EP (2013): Iron in infection and immunity. Cell Host Microbe 13: 509-519.

10. Crawford A, Wilson D (2015): Essential metals at the host-pathogen interface: nutritional immunity and micronutrient assimilation by human fungal pathogens. FEMS Yeast Res 15: fov071. 
11. Johnson EE, Wessling-Resnick M (2012): Iron metabolism and the innate immune response to infection. Microbes Infect 14: 207-216.

12. Niemiec MJ, De Samber B, Garrevoet J, et al. (2015): Trace element landscape of resting and activated human neutrophils on the sub-micrometer level. Metallomics 7: 996-1010.

13. Bystrzycka W, Moskalik A, Sieczkowska S, et al. (2016): The effect of clindamycin and amoxicillin on neutrophil extracellular trap (NET) release. Cent Eur J Immunol 41: 1-5.

14. Kalinowski DS, Richardson DR (2005): The evolution of iron chelators for the treatment of iron overload disease and cancer. Pharmacol Rev 57: 547-583.

15. Saliba AN, Harb AR, Taher AT (2015): Iron chelation therapy in transfusion-dependent thalassemia patients: current strategies and future directions. J Blood Med 6: 197-209.

16. Aleem A, Shakoor Z, Alsaleh K, et al. (2014): Immunological evaluation of beta-thalassemia major patients receiving oral iron chelator deferasirox. J Coll Physicians Surg Pak 24: 467-471.

17. Völlger L, Akong-Moore K, Cox L, et al. (2016): Iron-chelating agent desferrioxamine stimulates formation of neutrophil extracellular traps (NETs) in human blood-derived neutrophils. Biosci Rep 36: e00333.

18. Kono M, Saigo K, Yamamoto S, et al. (2016): Iron-chelating agent, deferasirox, inhibits neutrophil activation and extracellular trap formation. Clin Exp Pharmacol Physiol 43: 915-920.

19. Saigo K, Kono M, Takagi Y, et al. (2013): Deferasirox reduces oxidative stress in patients with transfusion dependency. J Clin Med Res 5: 57-60.

20. Iizuka M, Sagara R (2000): Fe(III)-IDA induces proliferation and sustains functions of various types of cultured cells as a substitute for transferrin. In Vitro Cell Dev Biol Anim 36: 495-501.

21. Hasan R, Rink L, Haase H (2013): Zinc signals in neutrophil granulocytes are required for the formation of neutrophil extracellular traps. Innate Immun 19: 253-264.

22. Urban CF, Ermert D, Schmid M, et al. (2009): Neutrophil extracellular traps contain calprotectin, a cytosolic protein complex involved in host defense against Candida albicans. PLoS Pathog 5: e1000639.

23. Kuzmicka W, Manda-Handzlik A, Cieloch A, et al. (2020): Zinc supplementation modulates NETs release and neutrophils' degranulation. Nutrients 13: 51.

24. Hasan R, Rink L, Haase H (2016): Chelation of Free Zn(2)(+) impairs chemotaxis, phagocytosis, oxidative burst, degranulation, and cytokine production by neutrophil granulocytes. Biol Trace Elem Res 171: 79-88. 